\title{
Respiratory disorders and neonatal outcomes of triplet pregnancies - our ten year experience
}

\author{
MONIKA LACHOWSKA ${ }^{1}$, KRZYSZTOF LACHOWSKI르, BARBARA KRÓLAK-OLEJNIK ${ }^{1}$
}

${ }^{1}$ Department of Neonatology, Wrocław Medical University, Poland

${ }^{2}$ Students' Scientific Club of Neonatology, Wrocław Medical University, Poland

Corresponding author:

Krzysztof Lachowski

Students' Scientific Club of Neonatology, Wrocław Medical University

Jan Mikulicz Radecki's University Hospital

ul. Borowska 213

50-556 Wroclaw, Poland

Phone: 0048605160594

E-mail:kplachowski@gmail.com

\section{ABSTRACT}

Objective. To compare respiratory disorders (respiratory distress syndrome, requirement for respiratory support, development of chronic lung disease), duration of hospitalization and other neonatal outcomes between newborns born from triplet pregnancies over a ten year period. Methods. A retrospective analysis of 34 triplet pregnancies delivered between 2006 and 2015 in one perinatal tertiary centre. Ninety-nine newborns from these pregnancies were divided into 2 groups: one consisted of 56 neonates (19 sets of triplets) born between 2006 and 2011 and the second contained 43 neonates delivered from 15 triplet pregnancies between 2012 and 2015.

Results. There were no differences in the incidence of respiratory distress syndrome and chronic lung disease between group I and group II. In both groups, a similar amount of patients required respiratory support. We did not notice any significant differences in the type of ventilation (mechanical ventilation or nasal continuous positive airway pressure -nCPAP), duration of ventilation, length of hospitalization or the incidence of complications of prematurity, such as 3rd or 4th grade intraventricular hemorrhage (IVH) and retinopathy of prematurity (ROP) stage > 2, between both groups.

Conclusion. Despite important progress in perinatal care and wide use of advanced technologies in neonatal intensive care there has been no significant improvement in neonatal outcomes of triplets during the past 10 years. Multiple pregnancies still remain a risk factor for respiratory disorders and other neonatal complications in prematurely delivered newborns.
Key words: triplets, newborn, respiratory disorders, outcome

\section{INTRODUCTION}

Over the past three decades a significant increase in the incidence of multiple gestations has been observed. This phenomenon is a consequence of widely used ART (artificial reproductive techniques) including induction of ovulation and in vitrofertilization (IVF) but also due to the trend of late maternity. (1-4) Multifetal gestation brings a higher risk of maternal complications during pregnancy and postnatal complications for the babies, which are usually born prematurely. The risk of preterm delivery for triplet pregnancies is approximately $90 \%$ while in twin gestations is more than $50 \%$, compared with only $10 \%$ of preterm births of single infants. $(5,6)$ The rate of preterm births before 32 weeks of gestation in triplets is 3.3-fold higher than in twins and 24.1-fold higher than in singletons. (1) A marked improvement of care for mothers with multiple pregnancies in specialized centers of perinatal care and early identification of risk factors for preterm delivery as well as significant progress in neonatal intensive care have contributed to increased survival and reduced morbidity of premature newborns. However, as shown in previous publications, it seems that this improvement is not significant in the case of children coming from triplet pregnancies. $(1,2)$

Therefore, the aim of our retrospective study was to compare respiratory disorders (respiratory distress syndrome, requirement for respiratory support, development of chronic lung disease), duration of hospitalization and other neonatal outcomes of triplet pregnancies over a 10 year period to detect any progress in this area and to compare the outcomes and the incidence of complications between the two groups of premature newborns: born from triplet pregnancies between 2006 to 2011 and between 2012 and 2015.

\section{MATERIAL AND METHODS}

This retrospective study was conducted in one tertiary perinatal center in Poland. The study population consists of 34 sets of triplets delivered over a period of ten years - between 2006 and 2015. Pregnancy history and neonatal data were obtained from medical records in the hospital database. Ninety-nine newborns from these pregnancies were divided into 2 groups: group I consisted of 56 live-born neonates (19 sets of triplets) born between 2006 and 2011 and group II consisted of 43 live-born infants delivered from 15 triplet pregnancies between 2012 and 2015. For both groups we analyzed the following parameters: maternal age, gestational age, birth weight, gender, 1st and 5th minute Apgar score as well as respiratory disorders like: respiratory distress syndrome (RDS), chronic lung disease (CLD), requirement and duration of respiratory support, type of respiratory support: invasive (mechanical ventilation via endotracheal tube) and non-invasive (nCPAP - nasal continuous positive airway pressure, SIPAP etc), surfactant administration and complications including retinopathy of prematurity (ROP) stage $>2$ and intraventricular hemorrhage (IVH) of III and IV grade. Duration of hospitalization and mortality rate were also compared. Gestational age (g.a.) was calculated from the data of the last menstrual period in 
spontaneously conceived pregnancies or from the data of embryo transfer for the IVF gestations. RDS was diagnosed based on clinical picture and chest x-ray. Surfactant was administered routinely to all infants born before 28th week of gestation, for infants delivered after 28th week g.a. only if they required intubation for RDS and if their oxygen requirement was more than 0.4. Chronic lung disease was defined as dependence on supplemental oxygen at the corrected g.a. of 36 weeks. Diagnosis of retinopathy of prematurity was raised by the consultant ophthalmologist after examination of the fundus. IVH was diagnosed by head ultrasound performed in all premature newborns in the neonatal invasive care unit (NICU).

For each parameter, the mean, median, standard deviation, lower and upper quartiles and range were calculated. Statistical significance between means for different groups was calculated by one-way analysis of variance (ANOVA), alternatively using the non-parametrical Kruskal-Wallis test, when the variances in groups were not homogeneous (the homogeneity of variance was determined by the Bartlett's test). Statistical significance between frequencies was calculated by the chi-square test with Yate's correction or if expected value was less than 5, by Fisher exact test. A p value of less than 0.05 was considered statistically significant. Statistical analysis was performed using EPIINFO Ver. 7.1.1.14 software package.

\section{RESULTS}

The mean gestational age for group I was $32.5 \pm 2$ weeks $(\mathrm{M} \pm \mathrm{SD})$ (min. 27 to $\max$. 35 weeks g.a.) and for group II was $32.7 \pm$ 1.3 weeks ( $\min .29$ to max. 34 weeks g.a.) ( $p=0.875$ as counted by Kruskal-Wallis test). There were also no significant difference in birth weight: $1727.3 \pm 404.1 \mathrm{~g}$ for group I ( $\min .730 \mathrm{~g}$, max. $2500 \mathrm{~g}$ ) vs. $1672.4 \pm 271.0 \mathrm{~g}$ for group II (min. 1090g, max. 2140g) (Kruskal-Wallis test $\mathrm{p}=0.342$ ). The two groups did not differ in terms of the type of respiratory support received (nCPAP vs invasive ventilation) - there were 21 infants ventilated by nCPAP and 12 by mechanical ventilation in group I in comparison to 20 and 9, respectively, in the second group. The chi-square test with Yate's correction was calculated, with a result of $\mathrm{X} 2=0,196$ and $\mathrm{p}=0.658$. The duration of respiratory support (group I: 12.3 \pm 14.0 days vs group II: $9.4 \pm 15.1$ days, reported values are $\mathrm{M} \pm \mathrm{SD}$ with $\mathrm{p}=0.432$ as counted by ANOVA test) and length of hospitalization $(26.4 \pm 16.2$ days for first vs. $30.7 \pm 13.9$ for second group, $\mathrm{M} \pm \mathrm{SD}$, ANOVA test $\mathrm{p}=0.176$ ) did not differ significantly between groups.

The mean mothers' age did not differ significantly between groups $(30.7 \pm 3.9$ for group I vs. $30.9 \pm 4.6$ for group II, ANOVA test $\mathrm{p}=0.785$ ). We did not observe a statistically significant difference between both groups in Apgar score at 1 minute $(6.85 \pm 1.63$ for first vs $6.84 \pm 1.72$ for sec- ond group, Kruskal-Wallis test $\mathrm{p}=0,900$ ), only at the 5th minute the Apgar score for group II was significantly higher (7.04 \pm 1.48 for group I vs $7.79 \pm 1.19$ for group II, $\mathrm{p}=0,0197$ as calculated by KruskallWallis test). Over the years, the incidence of severe respiratory insufficiency due to RDS (16 cases in first group vs. 6 cases in second group, the result of chi-square test with Yate's correction was $\mathrm{X} 2=2,22$ and $\mathrm{p}=0.136$ ) or CLD (6 cases in group I vs. 1 case in group II, Fisher test calculated $\mathrm{p}=0.134 \mathrm{~F}$ ) did not change significantly, as well as the incidence of intraventricular hemorrhage (III and IV grade) (7 cases in first group, 5 cases in second, the result of chi-square test with Yate's correction was $\mathrm{X} 2=0,032$ and $\mathrm{p}=0,858)$ and retinopathy of prematurity ( 5 cases in group I, 3 in group II, Fisher test $\mathrm{p}=0.999 \mathrm{~F}$ ). Three babies died in group I, while in group II one death was reported, which was not statistically significant (Fisher test $\mathrm{p}=0,631 \mathrm{~F}$ ) (table 1 ).

\section{DISCUSSION}

In the past decades, neonatology has become one of the most dynamically developing fields in medicine. Milestones such as prenatal corticosteroids for fetal lung maturation, intra-tracheal surfactant administration for RDS, new methods of ventilation including high frequency ventilation, non-invasive ventilation, inhaled nitric oxide (iNO) for the treatment of pul-

Table 1. Comparison of neonatal results of triplets over the 2 periods of time: 2006-2011 (group I) and 2012-2015 (group II).

\begin{tabular}{|c|c|c|c|}
\hline & $\begin{array}{l}2006-2011 \\
n=56\end{array}$ & $\begin{array}{l}2012-2015 \\
n=43\end{array}$ & p-value ( ${ }^{*}$ significant) \\
\hline Mean gestation age (weeks) & $32.5 \pm 2.0$ & $32.7 \pm 1.3$ & 0,875 \\
\hline Mean maternal age (years) & $30.7 \pm 3.9$ & $30.9 \pm 4.6$ & 0,785 \\
\hline Mean birthweight (grams) & $1727.3 \pm 404.1$ & $1672.4 \pm 271$ & 0,342 \\
\hline Apgar score at 1st minute & $6.86 \pm 1.63$ & $6.84 \pm 1.72$ & 0,900 \\
\hline Apgar score at 5 th minute & $7.04 \pm 1.48$ & $7.79 \pm 1.19 \quad \mathrm{p}=0.019$ & $0,0197^{\star}$ \\
\hline $\begin{array}{l}\text { Mean duration of respiratory sup- } \\
\text { port (nCPAP+MV) (days) }\end{array}$ & $12.3 \pm 14.0$ & $9.4 \pm 15.1$ & 0,432 \\
\hline $\begin{array}{l}\text { Mean length of hospitalization } \\
\text { (days) }\end{array}$ & $26.4 \pm 16.2$ & $30.7 \pm 13.9$ & 0,176 \\
\hline \multicolumn{4}{|l|}{ Respiratory support (n) } \\
\hline Ventilation in total & $33 / 56$ & $30 / 43$ & 0,266 \\
\hline nCPAP vs Invasive Ventilation & 21 vs $12 / 56$ & 20 vs $9 / 43$ & 0,658 \\
\hline BPD & 6 & 1 & $0,134 \mathrm{~F}$ \\
\hline IVH III/IV & 7 & 5 & 0,858 \\
\hline RDS & $16 / 56$ & $6 / 43$ & 0,136 \\
\hline ROP & 5 & 3 & $0,999 \mathrm{~F}$ \\
\hline Neonatal mortality & 3 & 1 & $0,631 \mathrm{~F}$ \\
\hline
\end{tabular}


monary hypertension, have improved the outcome of premature infants. However, as shown in previous publications this progress does not apply to triplets. $(1,2,7)$

Interestingly, despite the progress in prevention of preterm deliveries, mean gestational age at birth did not changed over the 10 -year period in our study, similar to other publications on triplet outcomes. $(1,2)$ The median gestational age at delivery for triplets is 32 weeks; by contrast twins have a mean age of delivery of 35.3 weeks and singletons 38.8 weeks. Multiple births have an increased risk of spontaneous preterm birth, but about $50 \%$ of them are cases of indicated preterm delivery due to maternal or fetal complications. (6-10)

According to Kaufman et al., triplets have similar outcomes as singleton and twins when stratified by g.a. with the only clinically significant difference being an increased incidence of ROP and mild IVH compared with singletons, but not when compared with twins. Other authors have made similar observations - neonatal outcome in multiple pregnancies depends mainly on gestational age. $(6,8,9)$ Nasseri et al. showed in his study that respiratory and non-respiratory outcomes between triplets and twins were not significantly different when matched with gestational age. (9) On the other hand, considering the long-term consequences of preterm delivery for multiplets they may be at increased risk for cerebral palsy (CP) and reduced cognitive function. (11)

Morency did not find any differences in neonatal outcomes between ART and spontaneous conceived triplets apart from an increase in RDS among ART triplets. (7) In the population of 370 preterm multiplets born $\leq 32$ weeks g.a. Shah found that the method of conception had no detectable effect on composite outcome defined as combination of death or any of the three neonatal morbidities (IVH grade 3 or 4, periventricular leukomalacia, ROP stage > 2 or CLD. (12)

According to Adegbite et al. perinatal outcome of spontaneously conceived triplet pregnancies depends on chorionicity. (13) The dichorionic triamniotic triplets had a 5.5-fold higher risk of adverse perinatal outcome (predominantly due to twin-to-twin transfusion syndrome and premature rupture of membranes) than trichorionic triamniotic gestations. In the study of Morency et al., monochorionicity was associated with an increase in adverse neonatal outcomes, specifically low birth weight, congenital abnormalities and neonatal mortality. (7) We could not compare this parameter in our study due to the lack of complete data in the obstetrics database. While advances in perinatal and neonatal care have improved neonatal outcomes, triplet and higher order pregnancies remain challenging to manage. (3) Special- ized perinatal care of triplet pregnancies in tertiary centers can give good neonatal outcomes for triplet gestations. (14) Although in many countries regulations on the number of embryos placed in utero during in vitro procedures have been introduced - there is no control on the number of embryos conceived through ovulation induction protocols. (1) Although fetoreduction is recommended in many Western countries, it may also be controversial for religious reasons (in the Middle East for instance). The main focus for reducing perinatal mortality and neonatal morbidity should be placed on strategies to prevent premature delivery and the rate of high order multiple pregnancies. (14)

Based on our observations and publications of other authors, we conclude that despite important progress in perinatal care and wide use of advanced neonatal intensive care technologies, there has been no significant improvement in neonatal outcomes of triplets during the past 10 years. Multiple pregnancies still remain a risk factor of respiratory disorders and other neonatal complications in prematurely delivered newborns. Both maternal and neonatal outcomes may be improved by antenatal and postnatal care in specialized tertiary centers of perinatal care.

\section{REFERENCES}

1. Weissman A, Ulanovsky I, Burke Y, Makroul IR, Blazer S, Drugan A. Triplet pregnancies - a three-decade perspective: do we fare better? Eur J Obstet Gynecol Reprod Biol. 2013;170:82-4.

2. Almeida P, Domingues AP, Belo A, Fonseca E, Moura P. Triplet pregnancies: perinatal outcome evolution. Rev Bras Ginecol Obstet. 2014;36:393-7.

3. Fennessy KM, Doyle LW, Naud K, Reidy K, Umstad MP. Triplet pregnancy: is the mode of conception related to perinatal outcomes? Twin Res Hum Genet. 2015;18:321-7.

4. Janvier A, Spelke B, Barrington KJ. The epidemic of multiple gestations and neonatal intensive care unit use: the cost of irresponsibility. J Pediatr. 2011;159:409-13.

5. Gardner MO, Goldenberg RL, Cliver SP, Tucker JM, Nelson KG, Copper RL. The origin and outcome of preterm twin pregnancies. Obstet Gynecol. 1995;85:553-7.

6. Blumenfeld Z, Abdallah W, Sela-Guttmann O, Brook OR. Triplet gestation - prevention, risks \& management dilemmas. Open Women's Health J. 2008;2:11-21.

7. Morency AM, Shah PS, Seaward PG, Whittle W, Murphy KE. Obstetrical and neonatal outcomes of triplet births - spontaneous versus assisted reproductive technology conception. J Matern Fetal Neonatal Med. 2016;29:938-43.

8. Kaufman GE, Malone FD, Harvey-Wilkes KB. Neonatal Morbidity and Mortality Associated With Triplet Pregnancy. Obstet Gynecol. 1998;91:342-8.

9. Nasseri F, Azhir A. The neonatal outcome in twin versus triplet and quadruplet pregnancies. J Res Med Sci. 2009;14(1):7-12.

10. Refuerzo JS. Impact of multiple births on late and moderate prematurity. Semin Fetal Neonatal Med. 2012;17:143-5.

11. Shinwell ES, Haklai T, Eventov-Friedman S. Outcomes of multiplets. Neonatology. 2009;95(1):6-14.

12. Shah V, Alwassia H, Shah K, Yoon W, Shah P. Neonatal outcomes among multiple births $\leq 32$ weeks gestational age: does mode of conception have an impact? A cohort study. BMC Pediatr. 2011;14(11):54.

13. Adegbite AL, Ward SB, Bajoria R. Perinatal outcome of spontaneously conceived triplet pregnancies in relation to chorionicity. Am J Obstet Gynecol. 2005;193:1463-71.

14. Chibber R, Fouda M, Shishtawy W, Al-Dossary M, Al-Hijji J, Amen A, et al. Maternal and neonatal outcome in triplet, quadruplet and quintuplet gestations following ART: a 11-year study. Arch Gynecol Obstet. 2013;288:759-67. 